\title{
ELECTRIC CURRENTS CONNECTED WITH THE PROTON \\ FLARES OF 7 JULY AND 2 SEPTEMBER, 1966
}

\author{
A. B. SEVERNY
}

Crimean Astrophysical Observatory, Nauchny, Crimea, U.S.S.R.

\begin{abstract}
It is observed that the change of the net magnetic flux associated with flares can exceed $10^{17} \mathrm{Mx} / \mathrm{s}$, which corresponds according to Maxwell's equation to the e.m.f. $\sim 10^{9} \mathrm{~V}$ which is specific for the high energy protons generated in flares. It is shown that this value of e.m.f. can hardly be compensated by e.m.f. of inductance which should appear due to the actually measured motions in a flare generating active region. The values of electric field strength thus found, together with measured values of electric current density (from $\operatorname{rot} H$ ), leads to an electric conductivity which is $10^{3}$ times smaller than usually adopted.
\end{abstract}

Figure 1 shows the behavior of the net magnetic flux

$$
\Phi=F_{s}-F_{n}
$$

connected with the proton flares of 7 July and 2 September, 1966. Values of $\Phi$ were obtained by planimetry of isogauss contour charts, an example of which is seen in Figure 2, for July 6 (Zvereva and Severny, 1970). The value $\Phi$ is measured for one and

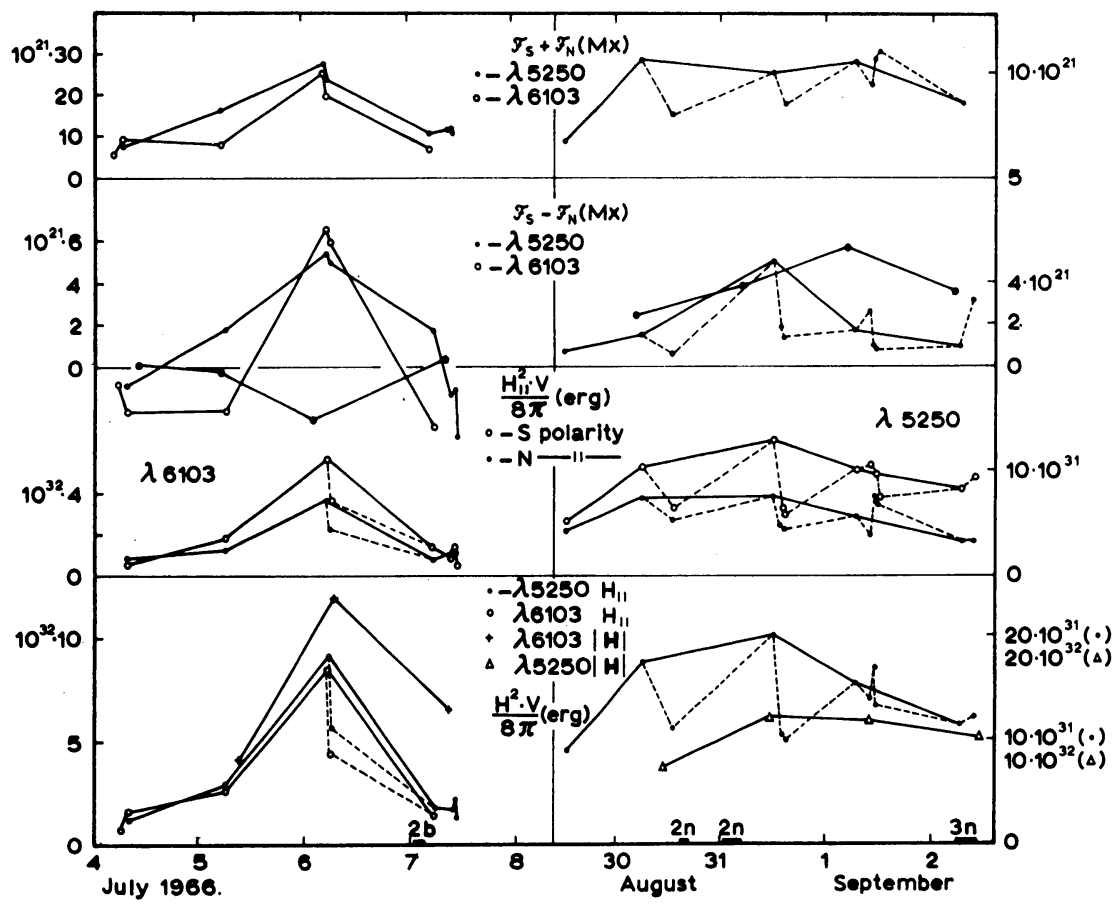

Fig. 1. The progressive change of the total flux $F_{\mathrm{S}}+F_{\mathrm{N}}$, net flux $F_{\mathrm{S}}-F_{\mathrm{N}}$ and of the energies of longitudinal $H$ (separately for $\mathrm{N}$-solid dots, and S-open circles polarities) and total $\mathbf{H}_{\|}$-fields associated with the flare of July 7, 1966 and of August 30 and September 2, 1966. Dashed lines in the later case are actual measurements, solid lines are drawn through the most reliable data (best seeing). 
the same fixed geometrical region. The boundary of this region is fixed to some extent arbitrarily just to include the main magnetic features. However an inspection of the whole series of maps shows that this is not important, because the main changes in $\Phi$ are connected with changes in position of isogauss lines inside this fixed region in
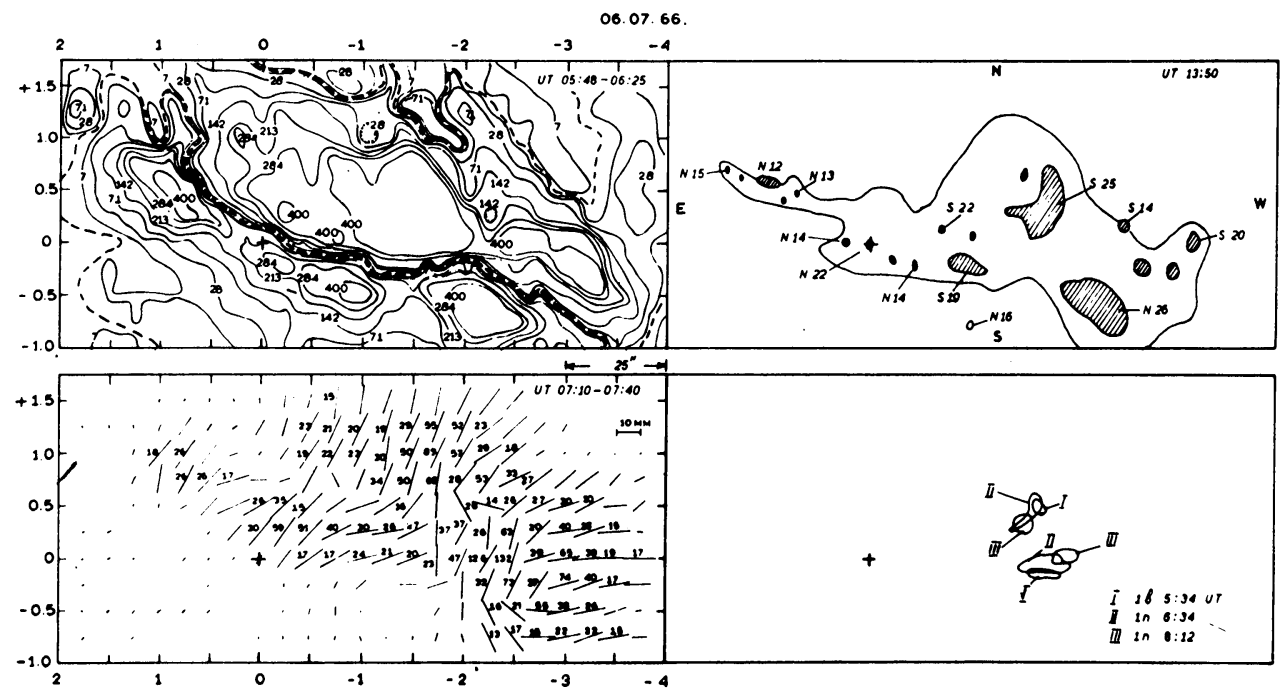

Fig. 2. An example of maps of longitudinal and transverse fields before the flare of July 7, 1966 (left), and the drawing of sunspots and flares (right) for the same area.

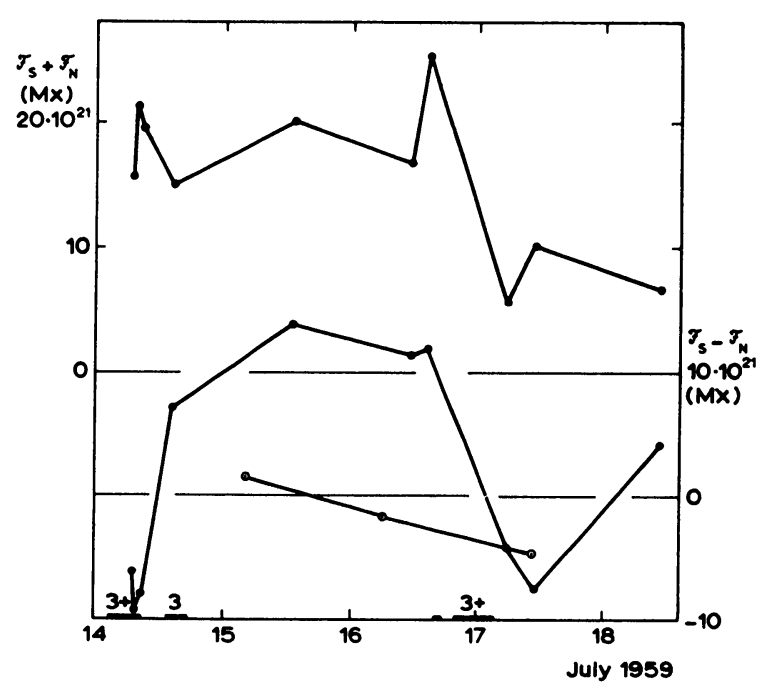

Fig. 3. The same as in Figure 1 but for the flare of July 16, 1959 (solid dots). Open circles are the values of the net flux from routine measurements of maximum fields in the umbrae of sunspots and their areas; the main contribution to the flux over the area is due to fields surrounding visible sunspots. 
the range of strong fields $(\geqslant 300-500 \mathrm{G})$, while the contribution from the periphery is negligible, not exceeding $10-15 \%$.

In both cases we have a bias of flux of one polarity before the flare ( $S$-polarity) which is characteristic of many other flares. A similar behavior of $\Phi$ shows also in the well known flare of 16 July 1959 as may be seen in Figure 3. A similar phenomenon for about 10 less important flares was found by Martres et al. (1968). For the flares under consideration we have Table I.

TABLE I

\begin{tabular}{lllllll}
\hline No. & Flares & Import & $\Phi$ (before) & $\Phi$ (after) & $\frac{\Delta \Phi}{\Delta t}$ & $\Delta t$ \\
\hline 1 & 17 July 59 & $3+$ & $+12 \times 10^{21} \mathrm{Mx}$ & $-8 \times 10^{21} \mathrm{Mx}$ & $21.75 \times 10^{17} \mathrm{Mx} / \mathrm{s}$ & 0. . $^{\mathrm{d}} 8$ \\
2 & 7 July 66 & $2+$ & $-2.0 \times 10^{21} \mathrm{Mx}$ & $+7.0 \times 10^{21} \mathrm{Mx}$ & $1.1 \times 10^{17} \mathrm{Mx} / \mathrm{s}$ & $1 . \mathrm{d}^{\mathrm{d}} 0$ \\
3 & 2 Sept. 66 & $2+$ & $+0.4 \times 10^{21} \mathrm{Mx}$ & $+4.3 \times 10^{21} \mathrm{Mx}$ & $0.33 \times 10^{17} \mathrm{Mx} / \mathrm{s}$ & $1 . .^{\mathrm{d} 25}$ \\
4 & 22 July 62 & 2 & $-1.0 \times 10^{21} \mathrm{Mx}$ & $-0.2 \times 10^{21} \mathrm{Mx}$ & $1.1 \times 10^{17} \mathrm{Mx} / \mathrm{s}$ & $1 .{ }^{\mathrm{h} 7}$ \\
\hline
\end{tabular}

Here we have added also the flares of 22 June 1962 and 17 July 1959 for which a set of maps before, during and after the flares were available (Howard and Severny, 1963; Severny, 1963). The values $\Delta \Phi / \Delta t$ in this table are in general agreement (a little higher) with (Martres et al., 1968). There is also a tendency for $\Delta \Phi / \Delta t$ to increase with the increase of importance of the flare.

It seems reasonable to adopt

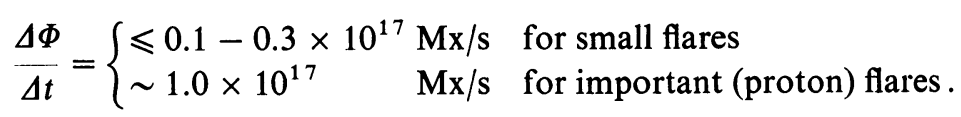

According to Maxwell's equation we have for the e.m.f.:

$$
\oint \mathbf{E}_{\mathrm{ds}}=-\frac{1 \Delta \Phi}{c \Delta t}=\begin{array}{ll}
(1-3) \times 10^{8} \mathrm{~V} & \text { small flares } \\
1.0 \times 10^{9} \mathrm{~V} & \text { important flares. }
\end{array}
$$

The following values for the electric field strength result (at $L=10^{10} \mathrm{~cm}$ for the length of a contour)

$$
E=\left\{\begin{array}{ll}
3.3 \times 10^{-4} & \text { small f. } \\
3.3 \times 10^{-5} & \text { large f. }
\end{array}\right\}(\text { CGSE) }
$$

This value corresponds closely to the energy of protons generated by flares, but this refers to a fixed contour, and the question arises whether this e.m.f. can really appear. It can, if the e.m.f. of inductance

$$
\frac{1}{c} \mathbf{V} \times \mathbf{H}
$$

can be disregarded as compared with the value $\mathbf{E}$ in the expression

$$
\mathbf{E}^{\prime}=E+\frac{1}{c} \mathbf{V} \times \mathbf{H}
$$


determining the e.m.f. through the moving contour, or if motions are too small. To evaluate the e.m.f., $1 / c(\mathbf{V} \times \mathbf{H})$, we have measured the motions in three different ways: (a) from the contraction of the region of $S$-polarity on isogauss-contours on the charts, (b) by the increase of the sizes of penumbra and umbra, (c) from the distances between the sunspots inside the group considered,(d) from instantaneous simultaneous measurements of line-of-sight velocities with the magnetograph when a group is not far from the limb.

The results are summarized in Table II.

TABLE II

Maximum velocities $(\mathrm{cm} / \mathrm{s})$

\begin{tabular}{lll}
\hline Method & Region $N$ & Region $S$ \\
\hline a & $0.85 \times 10^{4}$ & $1.05 \times 10^{4}$ \\
b S penumbra & 0.95 & 1.30 \\
$\quad$ S umbra & 1.20 & 0.80 \\
c & 0.50 & $\ldots$ \\
d & $\leqslant 1.50$ & $\leqslant 1.50$
\end{tabular}

So we have $v \leqslant 100-150 \mathrm{~m} / \mathrm{s}$ and

$$
\frac{1}{c} \mathbf{V} \times \mathbf{H} \leqslant 10^{-4}\left(=\frac{10^{4} \times 3 \times 10^{2}}{3 \times 10^{10}}\right)
$$

taking the field at the periphery of the region in question $=300 \mathrm{G}$ (actually it is a little less). The ratio:

$$
{ }_{c}^{1} v H / E=\left\{\begin{array}{ll}
<1.0 & \text { for small flares } \\
<0.1 & \text { for large flares }
\end{array}\right\} .
$$

If we take the isogauss line $10^{3}$ as the periphery, we should decrease $L$ by $5-10$ times; that means a 5-10 fold increase in $E$, and the ratio (3) becomes even smaller.

By the way, from the direct measurement of the field it follows that

$$
|\operatorname{rot} \mathbf{H}| \leqslant 1 \mathrm{G} / \mathrm{km}=10^{-5} \mathrm{G} / \mathrm{cm}
$$

and the current density is $\mathbf{j}=c / 4 \pi|\operatorname{rot} \mathbf{H}| \leqslant\left(3 \times 10^{10} / 1.26 \times 10\right) 10^{-5}=2.4 \times 10^{4} \mathrm{CGSE}$.

Hence from the equation

$$
\mathbf{j}=\sigma\left(\mathbf{E}+\frac{1}{c} \mathbf{V} \times \mathbf{H}\right)
$$

taking $E=3 \times 10^{-5}$ we obtain

$$
\sigma=\frac{\gamma}{E}=\frac{2.4 \times 10^{4}}{3 \times 10^{-5}} \simeq 10^{9} \mathrm{CGSE}
$$

which is $10^{3}$ times smaller than usually adopted, $\left(10^{12}\right)$ (cf. Kopecky and Kuklin, 1966).

The tendency for bright plages and flares to appear on the line $V_{\|}=0$ (Severny, 1960; 
Gopasyuk and Tsap, 1969) irrespective of whether they occur near the center or the edge of the disk, is also in favor of the considerations we have presented here. If further observations support our results we may consider the flare phenomenon as connected with strong electric currents and the corresponding mechanism can be the one presented by Alfvén and Carlquist in their theory of flares (Alfvén and Carlquist, 1967).

\section{References}

Alfvén, H. and Carlqvist, P.: 1967, Solar Phys. 1, 220.

Gopasyuk, S. and Tsap, T.: 1969, Astron. Zh. 46, 923.

Howard, R. and Severny, A.: 1963, Astrophys. J. 137, 1242.

Kopecky, M. and Kuklin, G.: 1966, Bull. Astron. Inst. Czech. 17, 45.

Martres, M., Michard, R., Soru-Iscovici, I., and Tsap, T.: 1968, Solar Phys. 5, 187.

Severny, A.: 1960, Izv. Krymsk. Astrofiz. Obs. 24, 281.

Severny, A. 1963, Izv. Krymsk. Astrofiz. Obs. 30, 161.

Zvereva, A. and Severny, A.: 1970, Izv. Krymsk. Astrofiz. Obs., in press.

\section{Discussion}

Sweet: In deriving the induction field it is necessary to observe the actual fluid velocity to an accuracy sufficient to compare it conclusively with the magnetic flux changes. It is not clear that you have attained this accuracy.

Severny: We estimated velocities, as I said in my talk, by four different methods - three of them are related essentially to the horizontal motions and one to the line of sight motions. All these bring us to the consistent result and values about $100 \mathrm{~m} / \mathrm{s}$. The same value comes out of measurements of line of sight velocities when the active area is near the border of the disk and when the sight-line velocity is essentially the velocity of horizontal motion. We think this estimate $100 \mathrm{~m} / \mathrm{s}$ is sufficiently reliable.

Maltby: Regarding the time scale involved in completing one magnetogram have you considered measuring a smaller area in order to look for short time scale variations?

Severny: The characteristic time-scale was determined by the interval between successive magnetograms which is $40^{\mathrm{m}}$. So we were concerned with the mean values for approximately this time-interval.

Harvey, J.: In order to compute the electric current from vector magnetograms, it is necessary to resolve the $180^{\circ}$ ambiguity inherent in transverse magnetic field measurements. What technique do you use to resolve this ambiguity?

Severny: This ambiguity can be avoided from the careful consideration how lines of force can go and how they cannot at a given position, and the strength of the 'sources' of lines of force - sunspots and magnetic hills. In most cases the discrimination is unambiguous.

Wiehr: As I pointed out yesterday, the flux disbalances are due to variations of the line profile from photosphere to spot, and they will disappear when using an unchanged line (e.g. Fe 6303 instead of $\mathrm{Fe} 5250$ ). I wonder, therefore, whether the net flux and moreover its changes have real physical meaning.

Severny: (1) Usually the main contribution to the flux comes not from the spots themselves but from the much bigger area around; moreover the contribution from umbrae can and must usually be disregarded due to their much smaller area and due to saturation effects inside sunspots. You should just do yourself the planimetry of isogauss magnetograph maps to realize this and compute the contribution of sunspots from the usual routine data. 